\title{
Correlations in Hot Dense Helium
}

\author{
Burkhard Militzer \\ University of California, Berkeley, Departments of Earth and Planetary Science and Astronomy, Berkeley, CA 94720, USA
}

(Dated: November 18, 2018)

\begin{abstract}
Hot dense helium is studied with first-principles computer simulations. By combining path integral Monte Carlo and density functional molecular dynamics, a large temperature and density interval ranging from 1000 to $1000000 \mathrm{~K}$ and 0.4 to $5.4 \mathrm{~g} \mathrm{~cm}^{-3}$ becomes accessible to first-principles simulations and the changes in the structure of dense hot fluids can be investigated. The focus of this article are pair correlation functions between nuclei, between electrons, and between electrons and nuclei. The density and temperature dependence of these correlation functions is analyzed in order to describe the structure of the dense fluid helium at extreme conditions.
\end{abstract}

\section{INTRODUCTION}

In the interiors of solar and extrasolar ${ }^{1}$ giant planets, light elements such as hydrogen and helium are exposed to extreme temperature $(\sim 10000 \mathrm{~K})$ and pressure $(10-1000 \mathrm{GPa})$ conditions. Shock wave measurements 22 are the preferred experimental technique to reach such conditions in the laboratory. Lasers $s^{3}$, magnetic fields $s^{4}$, and explosives $\frac{5}{5}$ have recently been used to generate shock waves that reached megabar pressures. The challenge of these experiments is first to reach such extreme conditions and secondly to characterize the properties of the material with sufficient accuracy so that planetary interior model actually can be improved. Single shock experiments yield the highest accuracy to the equation of state (EOS). However those can rarely reach densities much higher than 4 times the starting density, which means a large part of giant planet interiors cannot be probed directly $\underline{\underline{6} .7}$.

The limitation in density has recently been addressed by combining static compression in a diamond anvil cell with dynamic shock compression ${ }^{3}$. The experiments by Eggert et al. reached pressures of $200 \mathrm{GPa}$ in helium, which is a significant increase compared to the gas gun experiments by Nellis et al. $\stackrel{8}{\text { from }} 1984$ that had reached 16 GPa on the principal Hugoniot.

The properties of dense helium have also be the subject of a series of recent theoretical investigations with firstprinciples simulation techniques. $\mathrm{In}^{9}$, helium was predicted to reach 5.24 -fold compression in shock experiments. The compression ratio is larger than 4 because of electronic excitations that occur in the fluid at high temperature. Kietzmann et al $\stackrel{10}{\underline{10}}$ studied the rise in electrical conductivity using the Kubo-Greenwood formula and compared with results of shock-wave experiments by Ternovoi et al $\stackrel{11}{\underline{11}}$. Kowalski et al $\stackrel{12}{\underline{n}}$ studied dense helium in order to characterize the atmosphere of white dwarfs. The paper went beyond the generalized gradient approximation by considering hybrid functionals. Stixrude and Jeanloz ${ }^{13}$ studied the band gap closure in the dense fluid helium over a wide range of densities including conditions of giant planet interiors. Two recent studies of Jupiter's interior ${ }^{14.15}$, to very different extent, relied on a helium EOS derived the DFT-MD.

The insulator-to-metal transition in solid helium at high pressure was the subject of a recent quantum Monte Carlo study $\underline{16}$ that showed that standard density functional methods underestimate the band gap by $4 \mathrm{eV}$, which means the metallization pressure is underestimated by $40 \%$.

In this article, path integral Monte Carlo (PIMC) and density functional molecular dynamics (DFT-MD) simulations are combined to study pair correlation functions in fluid helium over a large density and temperature interval. This paper expands upon an earlier work ${ }^{17}$ that demonstrated that DFT-MD results at lower temperatures and PIMC data at higher temperatures can be combined into one coherent EOS table. Ref $\frac{17}{}$ also provide a free energy fit, computed adiabats and the electronic density of states. The EOS was compared with different semi-analytical free energy models $18,19,20$.

\section{METHODS}

Path integral Monte Carlo 21 is the most appropriate and efficient first-principles simulation techniques for quantum system with thermal excitations. Electrons and nuclei are treated equally as paths, although the zero-point motion of the nuclei as well as exchange effects are negligible for the temperatures under consideration. The Coulomb interaction between electrons and nuclei is introduced using pair density matrices that we derived using the eigenstates of the two-body Coulomb problem 22 . The periodic images were treated using an optimized Ewald break-up 23 that we applied to the pair action 24 . The explicit treatment of electrons as paths leads to the fermion sign problem, which requires one to introduce the only uncontrolled approximation in this method, the fixed node approximation 25,26 . We use the nodes from the free-particle density matrix and from a variational density matrix ${ }^{27}$. Besides this approximation, all 


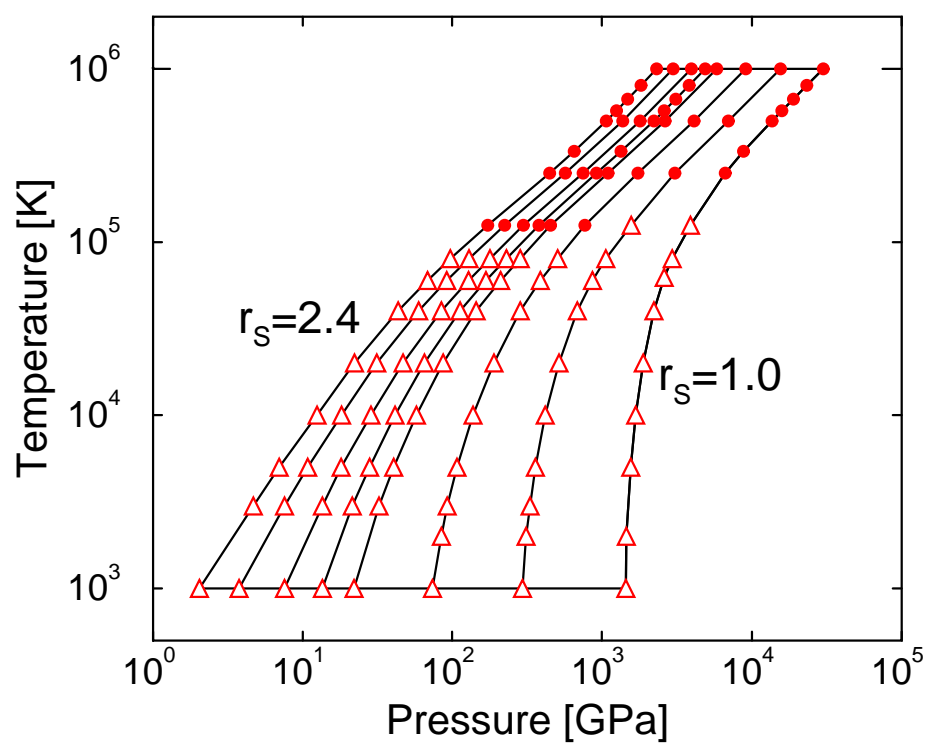

FIG. 1: Pressure-temperature diagram that indicates the conditions where PIMC (circles) and DFT-MD (triangles) simulations have been performed. The vertical lines show isochores for the following $r_{s}$ parameters: $2.4,2.2,2.0,1.86,1.75,1.5,1.25$, and 1.0 .

correlation effects are included in PIMC, which for example leads an exact treatment of the isolated helium atom. We performed PIMC simulations with 32 and 57 atoms. Additional details are given in reference $\underline{17}$.

The DFT-MD simulations were performed with either the CPMD code $\frac{28}{2}$ using local Troullier-Martins normconserving pseudopotentials 29 or with the Vienna ab initio simulation package ${ }^{30}$ using the projector augmented-wave $\operatorname{method}^{31}$. The nuclei were propagated using Born-Oppenheimer molecular dynamics with forces derived from either the electronic ground state or by including thermally excited electronic states when needed. Exchange-correlation effects were described by the Perdew-Burke-Ernzerhof generalized gradient approximation ${ }^{32}$. The electronic wavefunctions were expanded in a plane-wave basis with energy cut-off of 30-50 Hartrees. Most simulations were performed with $N=64$ using $\Gamma$ point sampling of the Brillioun zone.

\section{RESULTS}

Figure 1 shows the pressure-temperature-density conditions where PIMC and DFT-MD simulations have been preformed. The density will be discussed in terms of the Wigner-Seitz radius, $r_{s}$, that is defined by $V / N_{e}=\frac{4}{3} \pi\left(r_{s} a_{0}\right)^{3}$. There is a substantial difference in the slopes of the isochores in figure 1. At low temperature and high density, the isochores are nearly vertical because the dominant contribution to the pressure is provided by degenerate electrons. It takes a significant increase in temperature before thermal excitations of the electrons add in a noticible way to the pressure and for isochores to bend over to high pressures. At lower densities, the biggest contribution comes from the ionic motion, and one finds the typical pressure-temperature relation of a dense, but not degenerate fluid.

Figure 2 shows the nuclear pair correlation function, $g(r)$, derived from DFT-MD simulations,

$$
\begin{aligned}
& g(\mathbf{r})=\frac{V}{N(N-1)}\left\langle\sum_{i \neq j} \delta\left(\mathbf{r}-\left(\mathbf{r}_{\mathbf{i}}-\mathbf{r}_{\mathbf{j}}\right)\right)\right\rangle \\
& g(r)=\frac{V}{4 \pi r^{2} N(N-1)}\left\langle\sum_{i \neq j} \delta\left(r-\left|\mathbf{r}_{\mathbf{i}}-\mathbf{r}_{\mathbf{j}}\right|\right)\right\rangle,
\end{aligned}
$$

The sum includes all pairs of $N$ particles in volume $V=L^{3}$. By definition, $g(r)$ approached 1 for large $r$ in an infinite system. All simulations are performed in periodic boundary conditions and we only show results for $r \leq \frac{L}{2}$.

$1000 \mathrm{~K}$ is the lowest temperature under consideration. The simulations at the highest three densities froze during the course of the MD run. The simulations were started from scaled fluid configurations taken from simulations at lower densities. 


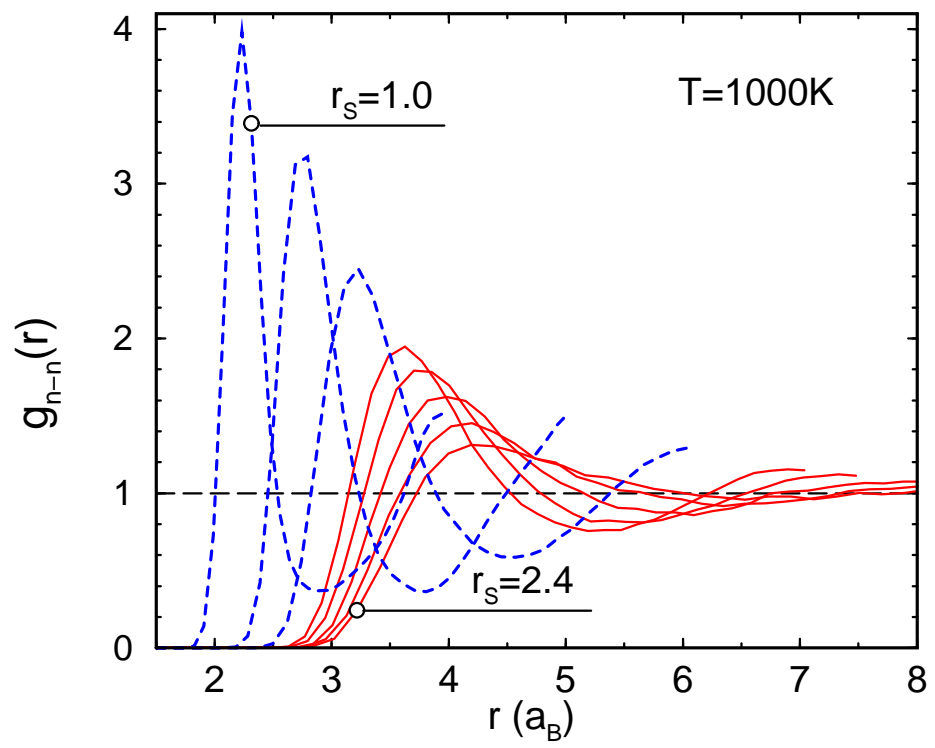

FIG. 2: Nuclear pair correlation functions are shown for the 8 different densities listed in figure 1 All results were obtained with DFT-MD simulations at a relatively low temperature of $1000 \mathrm{~K}$. The system at the the three highest density (dashed lines) froze into a solid during the course of the MD simulation. All simulations at lower densities (solid lines) remained in a liquid state.
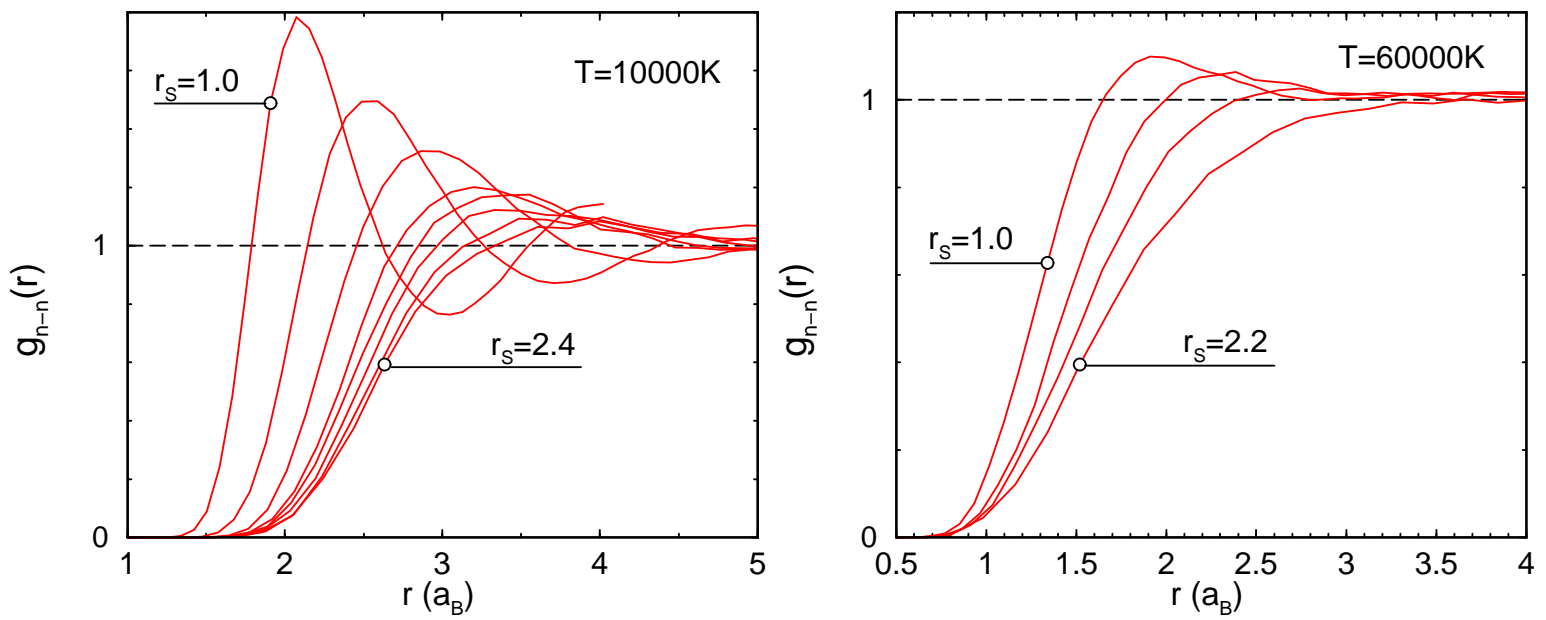

FIG. 3: Nuclear pair correlation functions computed with DFT-MD are compared for 10000 and $60000 \mathrm{~K}$. The left figure includes results for all densities shown in figure 1. For the right figure, only results for $r_{s}=1.0,1.25,1.5$, and 2.2 are shown for clarity.

Figure 2 shows a gradual decrease in the $g(r)$ oscillations with decreasing density. Such oscillations are the typical signature of the structure liquid or a solid. At high density, the $g(r)$ functions of both phases are more structured because the motion of the particles is more confined. The $g(r)$ functions are often fairly insensitive to the melting transition. The structure factor $S(k)$ and the diffusion constant are more reliable measures to identify melting in a simulation. However, that does not address the problem of superheating and supercooling that occurs in many simulations and also in nature. For an accurate determination of the melting temperature one needs to perform free energy calculations or perfrom simulations with liquid and solid in coexistence.

Figure 3 compares the nuclear pair correlation functions at two temperature of 10000 and $60000 \mathrm{~K}$. The increase in temperature leads to stronger collisions, which means the onset of the $g(r)$ is shifted to lower distances compared to $1000 \mathrm{~K}$. The motion of particles is less confined and, consequently, the peak height in the $g(r)$ is reduced substationally. At $60000 \mathrm{~K}$, the $g(r)$ shows only a small peak at the highest density, which then disappears altogether with decreasing density. 

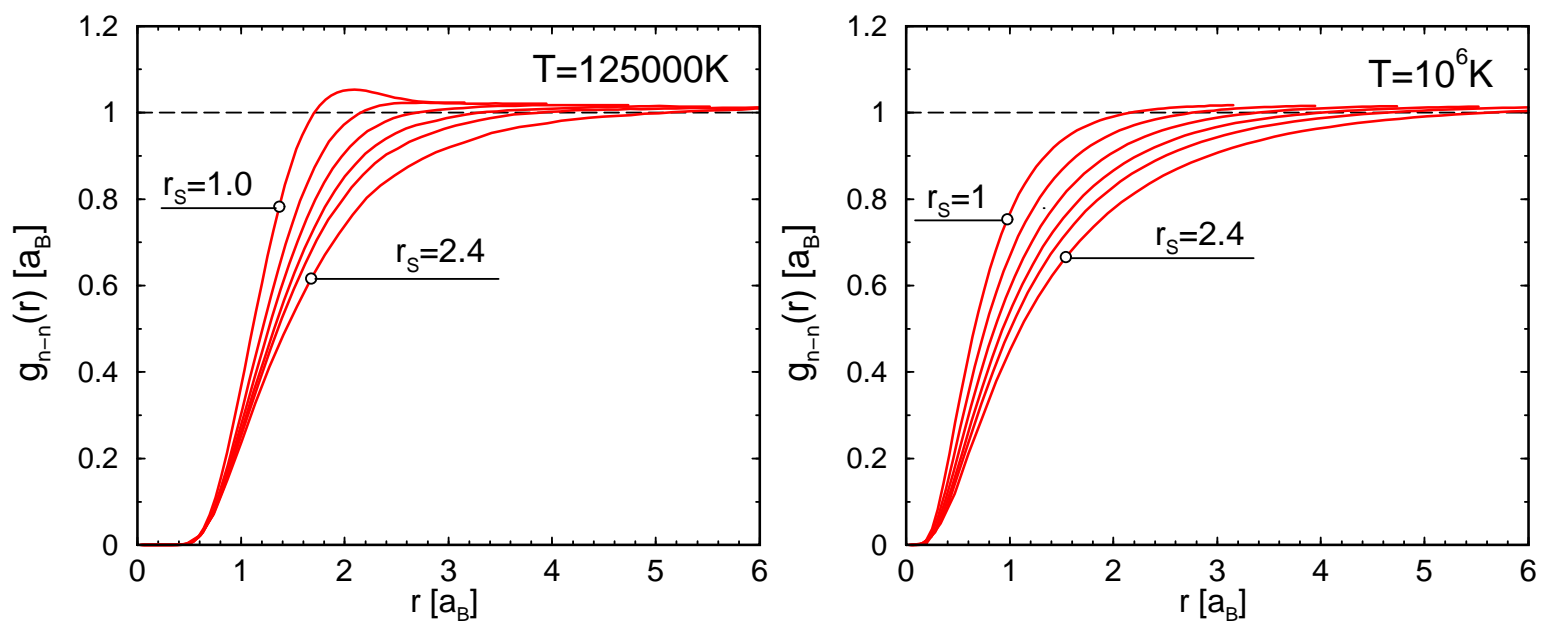

FIG. 4: Nuclear pair correlation functions computed with PIMC are shown for 125000 (left) and $10^{6} \mathrm{~K}$ (right) are shown for the following $r_{s}$ values: $1.0,1.25,1.5,1.75,2.0$, and 2.4 .
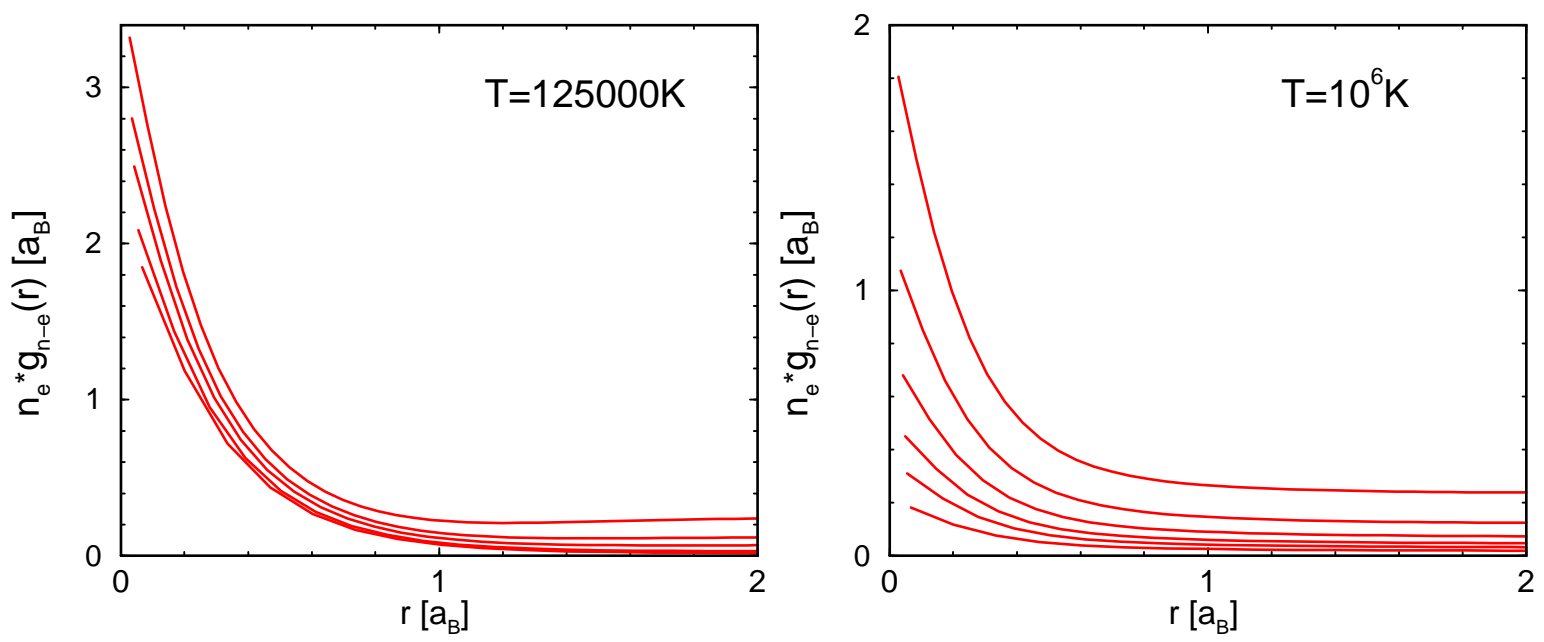

FIG. 5: Electron-nucleus pair correlation functions, $g_{n-e}(r)$, are shown for 125000 (left) and $10^{6} \mathrm{~K}$ (right) for the following $r_{s}$ values: 1.0 (top line) $1.25,1.5,1.75$ (only for $10^{6} \mathrm{~K}$ ) 2.0, and 2.4 (lowest line). The $g_{n-e}(r)$ function has been multilied by the volume density of electrons so that the area under the curves is related to the fraction of bound electrons.

Figure 4 shows the nuclear pair correlation function derived with PIMC for 125000 and $10^{6} \mathrm{~K}$. The expected trend continues; with increasing temperature and decreasing density the fluid becomes less structured. At an extreme temperature of $10^{6} \mathrm{~K}$, one can hardly find any positive correlation in the motion of the nuclei for the whole density range under consideration.

Figure 5 shows the correlation functions between nuclei and electrons. The $g_{n-e}(r)$ functions were multiplied by the electronic density, $N_{e} / V$, so that integral under the curve is related the number of electrons within a certain distance from a nucleus. This provides a qualitative estimate for the fraction of bound electrons. A quantititive estimate is difficult to give because unbound electrons in Rydberg scattering states also make contributions to $g_{n-e}(r)$ at small $r$. Furthermore, there is no straightforward way to tell to which nucleus a particular electron is bound. The long tail in the $g_{n-e}(r)$ includes unbound electrons as well as contribution from electrons bound to other nuclei nearby. In principle, the fraction of bound electrons can be determined from the natural orbitals of the reduced single particle density matrix ${ }^{33}$, but no practical method that is compatible with many-body simulations has been advanced. At very low density, however, approaches that fit $g(r)$ have worked reasonably well ${ }^{34}$.

At $125000 \mathrm{~K}$, the electron-nucleus pair correlation functions in figure [5]show a relatively weak dependence on density. The presence of the peak at small $r$ suggests that a fraction of the electrons are still bound at this temperature. At $10^{6} \mathrm{~K}$, the density dependence of the $g_{n-e}(r)$ is much stronger. At low density, there hardly any positive correlation 

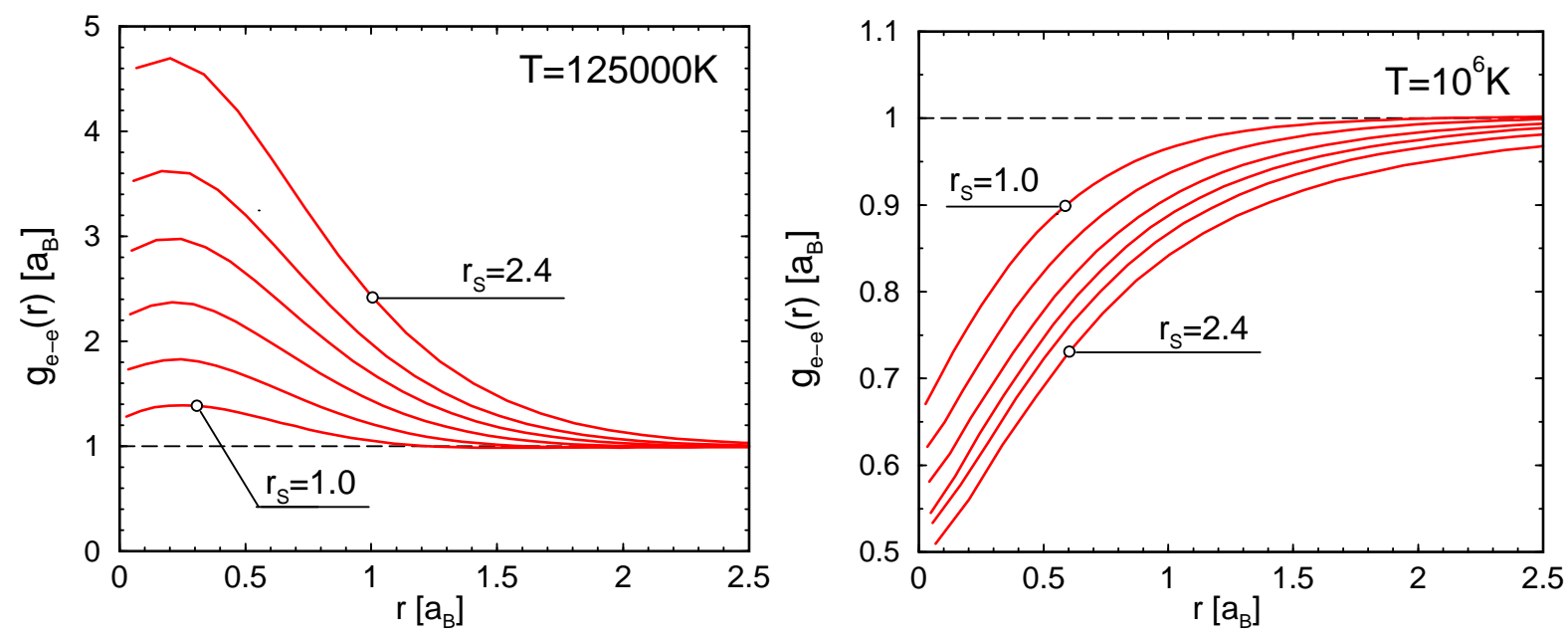

FIG. 6: The electron-electron pair correlation functions for electrons with opposite spins are shown for $125000 \mathrm{~K}$ on the left and for $10^{6} \mathrm{~K}$ on the right. The lines correspond to different densities represented by the following $r_{s}$ parameters: 1.0 , 1.25, $1.5,1.75,2.0$, and 2.4 .
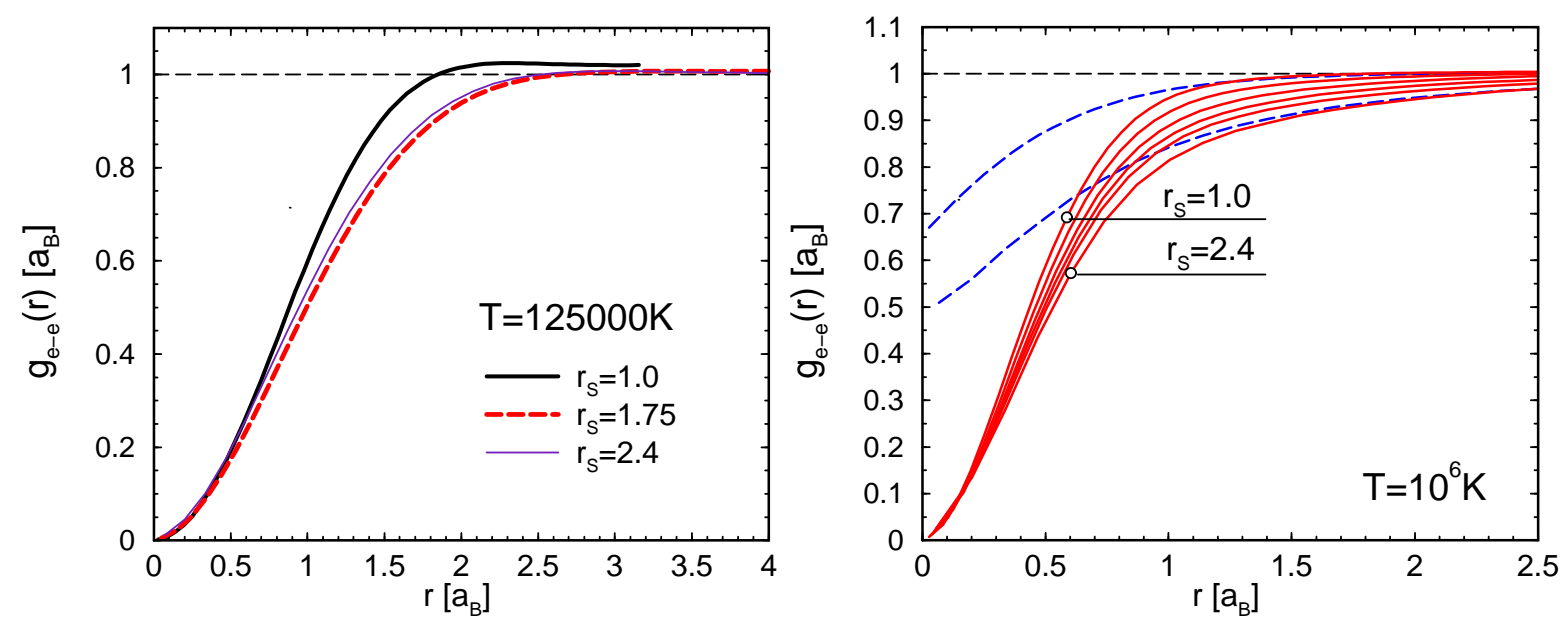

FIG. 7: The electron-electron pair correlation functions for electrons with parallel spins are shown for $125000 \mathrm{~K}$ on the left, and as solid lines (see figure 6 for $r_{s}$ values) for $10^{6} \mathrm{~K}$ on the right. The dashed lines on the right repeat correlation functions for electrons with opposite spins from figure 6 for $r_{s}=1.0$ and 2.4 .

in the motion of electrons and nuclei, which indicates the nuclei are almost fully ionized and most electrons are free. At higher densities, the motion of the electrons is more confined, which leads to an increased positive correlation with the nuclei.

The pair correlation function of the electrons with opposite spin in figure 6 shows a qualitatively different behavior at low and high temperature. Since they have different spins, the Pauli exclusion principle does not apply and only Coulomb repulsion keeps these pairs of particles apart. However, at $125000 \mathrm{~K}$, one observe a significant positive correlation, because two electrons with opposite spins are bound in a helium atom. So the peak in this $g_{e-e}(r)$ confirms the existance of bound electrons. The peak height decreases significantly with increasing density, which suggests that degeneracy in the electron gas increases and the number of bound states decreases accordingly. The slope of the isochores in figure 1 underlines the importance of the degeneracy at $125000 \mathrm{~K}$.

At $10^{6} \mathrm{~K}$ the behavior of the pair correlation function of the electrons with opposite spin is very different. The electrons are mostly unbound and are not degenerate. Coulomb repulsion leads to a negative correlation that much weaker than the Pauli exclusion for electron with parallel spin to be discussed next. With increasing density, the Coulomb repulsion has less of an effect, which means the value of $g(r=0)$ increases.

Figure 7 shows the pair correlation function of the electrons with parallel spins. The negative correlation at small $r$ is known as exchange-correlation hole. The effect of the Pauli exclusion is short-ranged, which can be understood 
from the graph at $10^{6} \mathrm{~K}$. At approximately $r=1.3 a_{0}$, the correlation functions of electrons with parallel and opposite spin converge, which marks the point where Pauli exclusion is no longer relevant.

The density dependence of the $g(r)$ of electrons with parallel spins at $125000 \mathrm{~K}$ is more difficult to interpret because of the presence of bound states. At $r_{s}=1$, one finds a small positive correlation, which can only be explained with a positive correlation in the nuclei $g(r)$ (figure 4) and the presence of bound electrons. Furthermore, one expects the size of the exchange-correlation hole would increase with decreasing density, which explains the difference between $r_{s}=1$ and 1.75. However, if the density is decreased further the size of the exchange-correlation hole shrinks slightly. Since there are more bound electrons present at $r_{s}=2.4$, the collision of the nuclei brings the electron closer together than they would be otherwise, which explains observed behavior.

In conclusion, the structure of a very simple fluid, helium, has been discussed at extreme pressure and density conditions. The observed trends are general and have relevance to other light elements are conditions of planetary interiors.

\section{Acknowledgments}

A part of the reported first-principles simulations were performed at NERSC and at NCSA.

\section{References}

1 A. Burrows. Nature, 433:261, 2005

${ }^{2}$ Y. B. Zeldovich and Y. P. Raizer. Academic Press, New York, 1966.

3 J. Eggert et al. Phys. Rev. Lett., 100:124503, 2008.

${ }^{4}$ M. D. Knudson, D. L. Hanson, J. E. Bailey, C. A. Hall, and J. R. Asay. Phys. Rev. Lett., 90:035505, 2003.

${ }^{5}$ V. E. Fortov et al. Phys. Rev. Lett., 99:185001, 2007.

${ }^{6}$ R. Jeanloz, P. M. Celliers, G. W. Collins, J. H. Eggert, K. K. M. Lee, R. S. McWilliams, S. Brygoo, and P. Loubeyre. Proc. Nat. Ac. Sci., 104:9172, 2007.

7 B. Militzer and W. B. Hubbard. AIP Conf. Proc., 955:1395, 2007.

8 W. J. Nellis et al. Phys. Rev. Lett., 53:1248, 1984.

9 B. Militzer. Phys. Rev. Lett., 97:175501, 2006.

10 A. Kietzmann, B. Holst, R. Redmer, M. P. Desjarlais, and T. R. Mattsson. Phys. Rev. Lett., 98:190602, 2007.

11 V. Ya. Ternovoi et al. AIP Conf. Proc., 620:107, 2002.

12 P. M. Kowalski, S. Mazevet, D. Saumon, and M. Challacombe. Phys. Rev. B, 76:075112, 2007.

13 L. Stixrude and R. Jeanloz. Proc. Nat. Ac. Sci., 105:11071, 2008.

14 B. Militzer, W. H. Hubbard, J. Vorberger, I. Tamblyn, and S. A. Bonev. in press Astrophys. J. Lett., 2008.

15 N. Nettelmann, B. Holst, A. Kietzmann, M. French, R. Redmer, and D. Blaschke. Astrophys. J., 683:1217, 2008.

16 S. A. Khairallah and B. Militzer. Phys. Rev. Lett, 101:106407, 2008.

17 B. Militzer. in press Phys. Rev. B, arXiv:0805.0317, 2009.

18 A. Förster, T. Kahlbaum, and W. Ebeling. Laser Part. Beams, 10:253, 1992.

19 D. Saumon, G. Chabrier, and H. M. Van Horn. Astrophys. J. Suppl., 99:713, 1995.

${ }^{20}$ Q. Chen, Y. Zheng, L. Cai, Y. Gu, and F. Jing. Phys. Plasmas, 14:012703, 2007.

21 D. M. Ceperley. Rev. Mod. Phys., 67:279, 1995.

22 E. L. Pollock. Comp. Phys. Comm., 52 :49, 1988.

${ }^{23}$ V. Natoli and D. M. Ceperley. J. Comp. Phys., 117:171-178, 1995.

24 B. Militzer and R. L. Graham. Journal of Physics and Chemistry of Solids, 67:2143, 2006.

25 D. M. Ceperley. J. Stat. Phys., 63:1237, 1991.

${ }^{26}$ D. M. Ceperley. Editrice Compositori, Bologna, Italy, 1996.

27 B. Militzer and E. L. Pollock. Phys. Rev. E, 61:3470, 2000.

28 CPMD, 1990-2006. Copyright IBM Corp, MPI für Festkörperforschung Stuttgart 1997-2001.

29 N. Troullier and J. L. Martins. Phys. Rev. B, 43:1993, 1991.

30 G. Kresse and J. Hafner, Phys. Rev. B 47, 558 (1993); G. Kresse and J. Hafner, Phys. Rev. B 49, 14251 (1994); G. Kresse and J. Furthmüller, Comput. Mat. Sci. 6, 15 (1996); G. Kresse and J. Furthmüller, Phys. Rev. B 54, 11169 (1996).

31 P. E. Blöchl. Phys. Rev. B, 50:17953, 1994.

32 J. P. Perdew, K. Burke, and M. Ernzerhof. Phys. Rev. Lett., 77:3865, 1996.

33 M. D. Girardeau. Phys. Rev. A, 41:6935, 1990.

34 B. Militzer, W. Magro, and D. Ceperley. Plenum Press, New York NY, 1998. 